\title{
Telescope of extreme ultraviolet (TEX) onboard SELENE: science from the Moon
}

\author{
I. Yoshikawa ${ }^{1}$, A. Yamazaki ${ }^{2}$, G. Murakami ${ }^{1}$, K. Yoshioka ${ }^{1}$, S. Kameda ${ }^{2}$, F. Ezawa ${ }^{1}$, \\ T. Toyota ${ }^{1}$, W. Miyake ${ }^{3 *}$, M. Taguchi ${ }^{4}$, M. Kikuchi ${ }^{4}$, and M. Nakamura ${ }^{2}$ \\ ${ }^{1}$ Department of Earth and Planetary Science, The University of Tokyo, 7-3-1 Hongo, Bunkyo, Tokyo 113-0033, Japan \\ ${ }^{2}$ Institute of Space and Astronautical Science, 3-1-1 Yoshinodai, Sagamihara, Kanagawa 229-8510, Japan \\ ${ }^{3}$ National Institute of Information and Communications Technology, 4-2-1 Nukuikita-machi, Koganei, Tokyo 184-8795, Japan \\ ${ }^{4}$ National Institute of Polar Research, 9-10-1, Kaga, Itabashi, Tokyo 173-8515, Japan
}

(Received April 16, 2007; Revised August 22, 2007; Accepted November 5, 2007; Online published April 9, 2008)

\begin{abstract}
The Upper Atmosphere and Plasma Imager (UPI) is to be launched in 2007 and sent to the Moon. From the lunar orbit, two telescopes are to be directed towards the Earth. The Moon has no atmosphere, which results in there being no active emission near the spacecraft; consequently, we will have a high-quality image of the near-Earth environment. As the Moon orbits the Earth once a month, the Earth will also be observed from many different directions. This is called a "science from the Moon". The two telescopes are mounted on a two-axis gimbal system, the Telescope of Extreme ultraviolet (TEX) and Telescope of Visible light (TVIS). TEX detects the O II $(83.4 \mathrm{~nm})$ and He II $(30.4 \mathrm{~nm})$ emissions scattered by ionized oxygen and helium, respectively. The targets of extreme-ultraviolet (EUV) imaging are the polar ionosphere, the polar wind, and the plasmasphere and inner magnetosphere. The maximum spatial and time resolutions are $0.09 \mathrm{Re}$ and $1 \mathrm{~min}$, respectively.
\end{abstract}

Key words: EUV, plasmasphere, polar wind, imaging, remote-sensing, SELENE.

\section{Introduction}

Standard theories on the polar wind indicate that only light ions such as $\mathrm{H}^{+}$and $\mathrm{He}^{+}$can overcome the terrestrial gravitational potential to escape from the polar ionosphere due to a pressure gradient along open field line and that the $\mathrm{O}^{+}$outflow is limited due to both its large mass and its loss by charge exchange with neutral $\mathrm{H}$ in the atmosphere (e.g., Axford, 1968; Banks and Holzer, 1968). Observations by polar orbiting satellites such as Dynamic Explorer 1 (DE-1) and AKEBONO (EXOS-D), however, found that the upward flux of $\mathrm{O}^{+}$is comparable to that of $\mathrm{H}^{+}$, especially under an active geomagnetic condition and during a period of a high solar activity (e.g., Chandler et al., 1991; Abe et al., 1993, 1996). Furthermore, GEOTAIL observed the cold dense $\mathrm{O}^{+}$flow of the ionospheric origin in the distant tail lobe (Seki et al., 1996, 1999, and references therein). The difference between the rate of $\mathrm{O}^{+}$outflow from the polar ionosphere and that of $\mathrm{O}^{+}$escape from the magnetosphere to interplanetary space indicates that there should be as-yet unknown transport processes for $\mathrm{O}^{+}$in the magnetosphere (Seki et al., 2001). The two-dimensional (2-D) O II imagery, which provides a global $\mathrm{O}^{+}$distribution, is expected to identify the transport routes and mechanisms of the cold $\mathrm{O}^{+}$ions and to reveal a quantitative balance between supply and loss, including thermal plasma.

\footnotetext{
*Now at Tokai University, 1117 Kitakaname, Hiratsuka 259-1292, Japan.

Copyright (c) The Society of Geomagnetism and Earth, Planetary and Space Sciences (SGEPSS); The Seismological Society of Japan; The Volcanological Society of Japan; The Geodetic Society of Japan; The Japanese Society for Planetary Sciences; TERRAPUB.
}

Our knowledge of the Earth's plasmasphere has increased steadily owing to a number of in-situ plasma measurements during the past several decades. The plasmasphere is filled with cold ions such as $\mathrm{H}^{+}, \mathrm{He}^{+}$, and a small amount of $\mathrm{O}^{+}$and is filled up to high density (about 100 ions $/ \mathrm{cm}^{3}$ ). The outer boundary, the plasmapause, is interpreted as a separatrix between closed and open convection trajectories (Nishida, 1966). Under a steady convection electric field condition, the plasmapause is considered to form a "teardrop" shape on the equatorial plane. The traditional models indicate that the plasmapause moves closer to the Earth when the large-scale convection electric field is enhanced. The plasma which was previously on a closed drift path then changes to an open drift path. As a result, the open drift path carries the plasma sunward, creating a plasmaspheric tail (Chen and Wolf, 1972) or a detected plasma region (Chappell, 1974). One question concerning the existence or nonexistence of such a structure has remained unresolved because the conventional in-situ observations for cold ions have an inherent shortcoming. The in-situ observations provide only local information, which does not include the necessary global aspect of the plasmasphere. It is therefore difficult to draw a definite conclusion on the above question. Global-scale observation is, therefore, a natural extension to many years of single-point in-situ observations. The primary motivation of this field of science is to visualize the global shape of the plasmasphere.

The remote-sensing methods using the extreme ultraviolet (EUV) emission of $\mathrm{He}^{+}$(He II $30.4 \mathrm{~nm}$ ) and $\mathrm{O}^{+}(\mathrm{O}$ II $83.4 \mathrm{~nm}$ ) have been expected to be a powerful tool to provide global perspectives on the escaping processes (Chiu et 
al., 1986; Meier, 1991) because conventional in-situ plasma particle measurement methods cannot detect plasma particles far from the satellite or thermal plasmas. The fundamental technology used to detect He II emission began with the rocket experiments in the 1970s and 1980s (Ogawa and Tohmatsu, 1971; Meier and Weller, 1972; Paresce et al., 1974; Chakrabarti et al., 1982). The measurements obtained using this technology clarified that the optical thickness of $\mathrm{He}^{+}$ions is very thin and that the intensity is very low (10 Rayleigh at most). They also clearly showed the feasibility of imaging the distribution of plasma around the Earth. In the 1990s, technological developments in EUV optics were reported in several articles (Cotton et al., 1993; Chakrabarti, 1993), and a breakthrough in the multilayer coating technique was first demonstrated by the sounding rocket experiment (Yoshikawa et al., 1997). The 2-D He II imaging of the terrestrial plasmasphere from the outside was subsequently accomplished by both the Planet-B (Nozomi) spacecraft (Nakamura et al., 2000; Yoshikawa et al., 2000a, 2001) and the Imager for magnetopause-toAurora Global Exploration (IMAGE) mission (Burch et al., 2001a, b; Sandel et al., 2001). Cold $\mathrm{He}^{+}$ions in the nearEarth plasma sheet were also optically observed by PlanetB (Yoshikawa et al., 2000b).

Optical observations on oxygen ions around the Earth have not yet been performed because of the difficulty in rejecting the bright $\mathrm{H} \mathrm{Ly}-\alpha$ line from the geocorona. We developed primitive equipment, denoted the eXtreme UltraViolet (XUV) sensor, that has thick indium film for the band-pass filter. The efficiency ratio of the O II emission to the $\mathrm{H} \mathrm{Ly}-\alpha$ line of this equipment is high enough to detect O II emission (Yamazaki et al., 2002). On December 4, 2000, this equipment flew onboard the sounding rocket SS-520-2 to investigate the mechanism of ion acceleration and/or heating at the cusp/cleft region. The XUV sensor successfully detected O II emission from the uppermost part of the polar ionosphere. The altitudinal variation from 150 through $1100 \mathrm{~km}$ indicated the existence of $\mathrm{O}^{+}$ions beyond the polar ionosphere and suggested that the $\mathrm{O}^{+}$ions energized in the cusp/cleft region may drift to the uppermost part of the polar ionosphere.

Using an upgrade version of this instrument we plan to obtain O II images of the polar wind from the lunar orbit with the SELenological and ENgineering Explorer (SELENE). The SELENE was launched by the H-IIA rocket in 2007 to be put into the orbit around the Moon. The aims of the SELENE project are to carry out scientific observations of the Moon, at the Moon, and from the Moon. The Upper atmosphere and Plasma Imager (UPI) on SELENE takes 2-D visible and extreme ultraviolet images of atomic and plasma distribution in the upper atmosphere and ionosphere and around the Earth. The component has two telescopes; one is Telescope for VISible light (UPI-TVIS), and the other is Telescope for EXtreme ultraviolet light (UPITEX). The UPI-TVIS imager detects four emission lines $(427.8,557.7,589.3,630.0 \mathrm{~nm})$ to simultaneously take auroral images around both of Earth's polar regions, and the UPI-TEX imager detects the resonance scattering emissions of oxygen ions (O II: $83.4 \mathrm{~nm}$ ) and helium ions (He II: $30.4 \mathrm{~nm}$ ) to take images of near-Earth plasmas. In this pa- per we present the design of the UPI-TEX imager and its calibrated performance.

\section{Science Target}

The UPI-TEX instrument provides global images of plasmas in the vicinity of the Earth by detecting He II (30.4 nm) emission scattered by $\mathrm{He}^{+}$ions and O II $(83.4 \mathrm{~nm})$ emission by $\mathrm{O}^{+}$ions. The observational targets are the plasmasphere, the polar wind, and the magnetosphere. In the following subsections, we present the scientific motivations and open questions which have not been resolved by past satellite observations.

\subsection{Global image of the plasmasphere}

The intensity of He II ( $30.4 \mathrm{~nm})$ emission from the main body of the plasmasphere is reported as about $5 \mathrm{R}$ by Nakamura et al. (2000). This result is consistent with early measurements of $1-10 \mathrm{R}$ done by sounding rockets from the inside-out view (e.g., Ogawa and Tohmatsu, 1971) and with simulated EUV images of the plasmasphere (e.g., Roelof et al., 1992). The intensity from the outer boundary of the plasmasphere has not been clarified yet by IMAGE mission, but reported to be in the range of 0.1-5 Rayleigh by the Planet-B satellite (Yoshikawa et al., 2000a). The intensity of $0.1-0.5 \mathrm{R}$ is considered to be a lower threshold level of the detection to grasp the global shape of the plasmasphere.

\subsection{Plasmasphere response to the interplanetary mag- netic field (IMF)}

Murakami et al. (2007) used the sequential EUV images of the plasmasphere obtained by NASA's IMAGE satellite and investigated the plasmaspheric response time to the solar wind electric field that was measured by solar monitor (ACE satellite). They showed that the plasmapause response to the southward turning of the IMF takes 10$30 \mathrm{~min}$, with an average of $18 \mathrm{~min}$. Their result is consistent with the time scale derived from the ionospheric observations on ground stations (Ridley et al., 1998; Ruohoniemi and Greenwald, 1998; Lu et al., 2002; Kikuchi et al., 1996; Hashimoto et al., 2002). Based on this accordance of data, these researchers concluded that the electric field penetrates from the magnetopause to the inner magnetosphere through the ionosphere. However, they also insisted that-due to limited EUV data, they found only 16 events-the result should be re-investigated using a more completed data set of EUV images. The IMAGE satellite orbits near the Earth. As such, it cannot always monitor the whole plasmasphere, and there is always an interval of at least a few hours between images, which makes it difficult to estimate the timelag between the IMF arrival at the magnetosphere and plasmasphere evolution. TEX observation improves this difficulty because SELENE provides the best platform to command a panoramic view of the whole plasmasphere. As cited above, the intensity of $0.1-0.5 \mathrm{R}$ is a lower threshold level to grasp the plasmaspheric response.

\subsection{Net escape rate of Earth's oxygen to space}

The observed polar outflow rate for $\mathrm{O}^{+}$ions corresponds to the loss of about $18 \%$ of the present-day atmospheric oxygen over 3 billion years. However, part of this apparent loss can actually be returned to the atmosphere. Examining loss rates of four already identified escape routes with highaltitude spacecraft observations, Seki et al. (2002) showed 
that the total oxygen loss rate inferred from current knowledge is about one order of magnitude smaller than the polar $\mathrm{O}^{+}$outflow rate. They then suggested that there may be a substantial return flux from the magnetosphere to the low-latitude ionosphere and also implied that this may indicate the existence of an as-yet unknown loss process for terrestrial oxygen. Otherwise, the net escape loss over 3 billion years may drop to about $2 \%$ of the current atmospheric oxygen content. This issue is very important not only for magnetosphere physics but also for the field of planetology.

Photometric observation is a very useful approach to resolve this issue as it is difficult to observe the escape of cold $\mathrm{O}^{+}$ions by in-situ measurements at energies below $50 \mathrm{eV}$ without spacecraft potential control (Seki et al., 2002).

\subsection{Continuous leakage of plasmaspheric materials}

Recent studies have revealed another feature of the plasmasphere. According to in-situ plasma measurements carried out on multiple geosynchronous satellites, the standard teardrop model of the plasmasphere, where the bulge size and location are determined by the convection electric field strength, is not a completely comprehensive description even under low geomagnetic activity (Moldwin et al., 1994). Furthermore, Matsui et al. (1999) have reported that GEOTAIL on a higher $\mathrm{L}$ value path $(\mathrm{L} \sim 10)$ often found plasmaspheric materials independent of $\mathrm{Kp}$. These researchers also concluded that cold plasma continuously convects toward the magnetopause. These results force us to verify the classical understanding and morphology of the plasmasphere/plasmapause that were constructed in the 1960 s.

According to Matsui's statistical analysis, the structure of cold ions along the GEOTAIL path $(\mathrm{L}>10)$ is quasi-stable, with a density of $2-10 \mathrm{~cm}^{-3}$. If we assume that $10 \%$ of such a population consists of $\mathrm{He}^{+}$ions, that they spread along the dusk-to-dawn direction of $10 \mathrm{Re}$, and that the g-factor of He II (30.4 nm) emission is $2 \times 10^{-5}\left(\right.$ ion $\left.^{-1} \mathrm{~s}^{-1}\right)$, then the intensity of $\mathrm{He}$ II $(30.4 \mathrm{~nm})$ from this source is estimated at $0.026-0.13 \mathrm{R}$. Thus, it is necessary to construct optics with a lower threshold level of about $0.02 \mathrm{R}$ to identify this unique phenomenon.

\subsection{Plasmasphere refilling}

The refilling of the plasmasphere has attracted considerably theoretical interest for several decades (see "Plasmasphere Refilling" special section in Journal of Geophysical Research, 97(A2), 1047-1179, 1992). Scientifically important studies on plasmasphere refilling were carried out in the 1960s and early 1970s. These were observational (Park, 1970; Chappell et al., 1971) and theoretical and/or modeling studies (Banks et al., 1971; Schulz and Koons, 1972; Chen and Wolf, 1972; Grebowsky, 1971) that addressed various refilling problems. One of the unsolved and highlighted issues is how plasma distribution approaches a diffusive equilibrium state under the condition of refilling, where plasma is supplied from the conjugate ionospheres through field-aligned supersonic flows. Most models dealing with refilling of the plasmasphere rely on initial conditions, such as plasma density, flow velocity, and temperature, in an empty flux tube. However, there is no theoretical basis to decide on the initial distributions, nor is there firm guidance from the observations. Because of the scarcity of information, the initial conditions are arbitrarily chosen in the models. The choice is generally only based on the fact that the equatorial plasma density in a depleted flux tube is as low as $1 \mathrm{~cm}^{-3}$.

We do not know how this low equatorial density connects with the high ionospheric density. In this regard, systematic observation along a depleted flux tube should reveal the subsequent refilling process. It is impossible to carry out such observations, however, using a single satellite, because it is impossible to know latitudinal and longitudinal distributions of plasma simultaneously.

The EUV imaging is a promising methodology to overcome this difficulty. Based on the theoretical considerations, a time resolution of $30 \mathrm{~min}$ is necessary to examine the refilling issue (Wilson et al., 1992). The measured He II $(30.4 \mathrm{~nm})$ intensity of the refilling trough region is $0.02-$ $0.1 \mathrm{R}$ as cited above. TEX should achieve this capability of time and spatial resolutions.

\subsection{Inconsistency of old and new topics on the plasma- sphere}

The subject of the continuous leakage of cold plasma introduced in Section 2.4 may be related to the issue of plasmasphere refilling. On one hand, in recent studies, such as that of Matsui, it has been possible to determine the rate of ion leakage from the plasmasphere provided that the amount of plasma supplied directly from the ionosphere is not dominant. On the other hand, such as in studies of the plasmasphere refilling, it is difficult to determine the evolution of the content in the same flux tube from the in-situ observation, because a flux tube drifts regardless of a satellite orbital motion. The early measurements on the refilling rate of the ionospheric plasma may include the contribution of plasma leakage from the inner plasmasphere. Consequently, the refilling rate may be overestimated. The EUV observation provides promising clues for the verification of the above two processes-i.e., the plasmasphere refilling and continuous leakage of plasmaspheric ions.

\subsection{Cold ions in the plasma sheet}

It is a well known fact that the plasma sheet is mainly populated with hot $(\mathrm{keV}) \mathrm{H}^{+}$and $\mathrm{O}^{+}$ions and involves a small amount of $\mathrm{He}^{+}$ions. The polar wind is the most substantial source flux of low-energy ions entering the nearEarth plasma sheet (XGSM>-30 Re). The cold plasma in the polar wind is energized up to several $\mathrm{keV}$ at its entry to the plasma sheet by a number of possible mechanisms (Lyons and Speiser, 1982; Cowley, 1980; Ondoh and Aikyo, 1986; Saito et al., 1994). There is an open question on plasma density which cannot be answered with current data. Chappell et al. (1987) estimated the average density of $\mathrm{He}^{+}$in the plasma sheet on the basis of the outflow flux of $\mathrm{He}^{+}$ions in the polar region: $0.007 \mathrm{~cm}^{-3}$ at quiet times and $0.035 \mathrm{~cm}^{-3}$ at active times. Their numbers exceed a density of $0.005 \mathrm{~cm}^{-3}$ based on in-situ measurements (Lennartsson and Shelley, 1986), and these researchers that the difference is due to the existence of an invisible lowenergy plasma $(<10 \mathrm{eV})$ that has never been observed because spacecraft are typically charged to positive potentials exceeding ion energy (Chappell et al., 1987). These estimations correspond to a column density of $1.3-6.7 \mathrm{E} 8 \mathrm{~cm}^{-2}$ from the dusk-to-dawn view of a 30-Re optical length. If a 
typical g-factor of $2 \mathrm{E}-5\left(\mathrm{~s}^{-1}\right.$ ion $\left.^{-1}\right)$ is assumed, the intensities of He II $(30.4 \mathrm{~nm})$ are calculated as $0.003-0.013 \mathrm{R}$. Yoshikawa et al. (2000b) also reported that a signal up to $0.02 \mathrm{R}$ was detected from the outside of the plasmasphere, but due to a low spatial resolution of the instrument, they could not completely identify the origin. TEX observation on the lunar orbiter surely identifies the origin.

A study of this topic does not need a high time-resolution EUV image. The photometric measurement of the plasma sheet with a detection capability of $0.01-0.02 \mathrm{R}$ with the spatial resolution of $3 \mathrm{Re}$ should bring a definite conclusion on it.

\subsection{Cold ions in the distant tail lobe/mantle region}

The plasma transport process from the ionosphere to the distant magnetotail has been one of the most important issues of geophysics in space. After the GEOTAIL satellite revealed that ions of ionospheric origin $\left(\mathrm{O}^{+}\right.$and $\mathrm{He}^{+}$) exist over a wide range of the tailward distance in the lobe/mantle regions up to $200 \mathrm{Re}$ from the Earth (Mukai et al., 1994; Hirahara et al., 1996), there has been much debate on the transport mechanism-i.e. what prevents ionospheric plasma from descending into the plasma sheet before reaching at such a large distance? Seki et al. (1998) proposed three plausible sources of survival ions in the distant tail lobe/mantle regions: (I) dayside polar ion outflow; (II) plasmaspheric materials stripped off the plasmasphere; (III) energetic upward flowing ions (UFIs) from the nightside auroral oval. (It is noted that they examined only $\mathrm{O}^{+}$ ions, but their argument is also valid for $\mathrm{He}^{+}$ions.) The EUV imaging will play a crucial role in determining the source region.

We have already referred to the intensities from sources (I) and (II). The expected intensity from source (I) is considered to be the same as that from the dayside refilling trough region, shown in Section 2.6. The intensity from source (II) also has been considered in Section 2.4. As for the other candidate of the energetic UFIs, it has been reported that the UFIs consist primarily of $\mathrm{H}^{+}$and $\mathrm{O}^{+}$ions and have fluxes of 1E6 to 1E8 (ions s $\mathrm{sm}^{-1}$ ) over the auroral oval at an altitude of $1000 \mathrm{~km}$ (Yau et al., 1985). There have been only a few studies on $\mathrm{He}^{+}$ions in the UFIs, but the upper limit of $\mathrm{He}^{+}$ions has been reported to be $5 \%$ of the total flux at the most (Collin et al., 1984). If we assume an energy of $100 \mathrm{eV}$, the density of $\mathrm{He}^{+}$ions in the UFIs ranges between 0.01 and $1 \mathrm{~cm}^{-3}$. Therefore, the intensity of the UFIs above the auroral oval is estimated to be $2 \mathrm{E}-6$ to $2 \mathrm{E}-4 \mathrm{R}$. Source (III) seems to be difficult to identify using our instrument, while the other two candidates are recognizable.

On the other hand, based on the results of investigations of lobe/mantle regions by GEOTAIL, the typical density of $\mathrm{He}^{+}$is $0.005 \mathrm{~cm}^{-3}$ or less, the temperature is approximately $300 \mathrm{eV}$, and the tailward velocity is $200 \mathrm{~km} \mathrm{~s}^{-1}$ (e.g., Seki et al., 2000). We estimate the intensity in distant lobe/mantle regions to be $7.5 \mathrm{E}-5 \mathrm{R}$, assuming an optical length of $60 \mathrm{Re}$ along the dusk-to-dawn direction. Thus, a detectable limit of $1 \mathrm{E}-5$ to $1 \mathrm{E}-4 \mathrm{R}$ is needed for a complete understanding of the topology of the terrestrial cold ion flow.

\subsection{Short- and long-terms variation of $\mathrm{O}^{+}$outflow}

Previous studies reported that the occurrence of the $\mathrm{O}^{+}$ outflow event depends on the direction of the IMF, the geomagnetic activity, the seasonal condition, and the solar activity (e.g., Chandler et al., 1991; Abe et al., 1993, 1996). Chiu et al. (1990) predicted the intensity of $\mathrm{O}^{+}$outflow to range from 0.3 to $1 \mathrm{R}$. Recently the Low-Energy Neutral Atom imager (LENA) on IMAGE observed the oxygen ion burst outflow in response to an enhancement of the dynamic pressure of the solar wind at the moment of the passage of a shock produced by a coronal mass ejection (Moore et al., 2001; Fuselier et al., 2001). These observations indicated that the $\mathrm{O}^{+}$outflow flux has both a long- and short-term variation; therefore, a constant monitoring and simultaneous observation of solar wind plasma are necessary to identify $\mathrm{O}^{+}$outflow mechanisms. The LENA imager can detect the energetic neutral atoms within the energy range of 10 $750 \mathrm{eV}$ (Moore et al., 2000). The TEX instrument, however, can detect the $\mathrm{O}$ II emission scattering by the thermal $\mathrm{O}^{+}$as well as the low energy ions, i.e., it can measure $\mathrm{O}^{+}$ with a thermal energy range at which ions will return to the atmosphere due to the gravitational force once they flow upward. The TEX instrument observes during an isometric orbit from the Earth and is able to detect the short-term variation more easily than the IMAGE mission.

\subsection{Thermal oxygen ion distribution in magneto- sphere}

Thermal $\mathrm{O}^{+}$observation is not performed in the inner magnetosphere because the existence of the high energetic particles in the radiation belt contributes the large noise count level. The high-energy electrons at the outer radiation belt inject inward during the geomagnetic storm (Obara et al., 2000), and the thermal plasma should inject inward together and precipitate to the ionosphere at the low latitude. Furthermore, the oxygen ions flux between supply and loss in the magnetosphere does not keep in balance (Seki et al., 2001). We suggest that these problems arise from the lack of any observations of the thermal plasma distribution in the high-energy plasma environments of the magnetosphere. If the O II optical observation is successfully performed, the distribution of the thermal $\mathrm{O}^{+}$will constantly obtained, and its behavior and its quantity balance will be elucidate.

Our key technology to clarify the above issues is to build bright optics in the spectral range of EUV. A multilayercoated mirror that has a high reflectivity in the EUV range is of particular importance (Yoshikawa et al., 1997). We have already demonstrated the capabilities of instruments with these technologies by the Planet-B mission (Yoshikawa et al., 2001). The success of the Planet-B experiment has motivated us to attempt continuous global EUV imaging.

\section{Instrumentation}

Effective imaging of the near-Earth plasma distribution requires global 'snapshots' with wide field-of-view (FOV). Lunar orbiter (SELENE) provides us with an ideal platform that commands a panoramic view of the near-Earth situation. Our telescope of EUV is mounted on a two-axis gimbal system together with Telescope of Visible light (TVIS), as shown in Fig. 1. The pointing accuracies of the equatorial mounting are 3.24" around the Azimuthal and 2.68" 


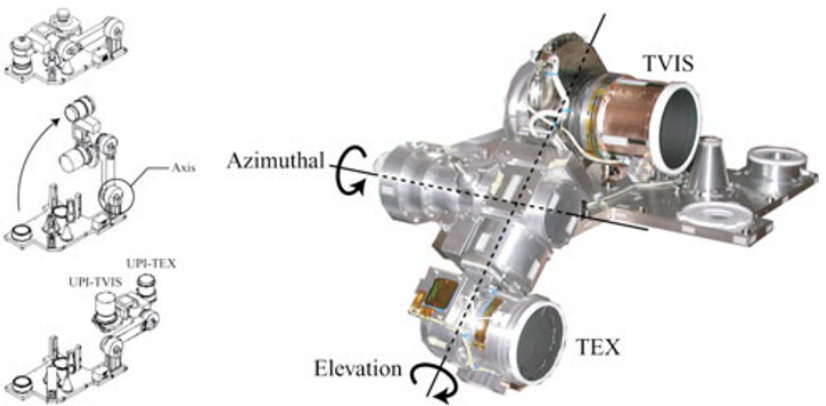

Fig. 1. Upper atmosphere and Plasma Imager (UPI) aboard SELENE satellite. Two telescopes are installed on the equatorial mounting (motor-controlled azimuthal and elevation axes) gimbal. After the insertion to the lunar orbit, the system is open (left panels) in order to make the two telescopes ready to point to the Earth. Pointing accuracy of the equatorial mounting is $3.24^{\prime \prime}$ around Azimuthal and 2.68" around Elevation axis.

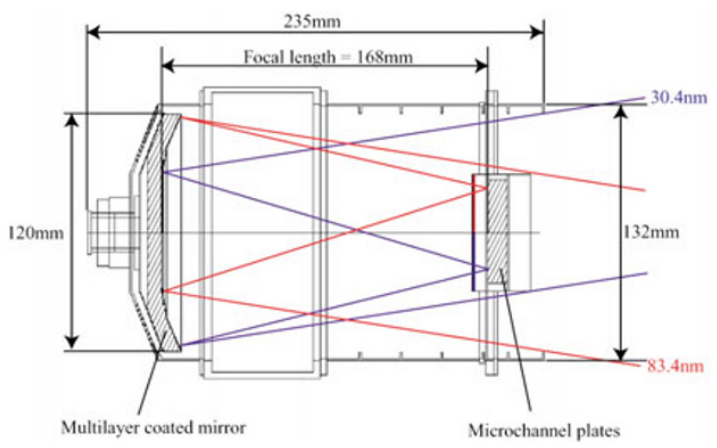

Fig. 2. Configuration of Telescope of Extreme ultraviolet (TEX) boarded on the SELENE satellite. Main optical components are a multilayer coated mirror, a split metal thin filter, microchannel plates. Field-of-view is separate, O II (83.4 nm, red line in the figure) and He II (30.4 nm, blue line) areas.

around the Elevation axes. These correspond to the spatial resolution of $5 \mathrm{~km}$ on the Earth's surface.

The UPI-TEX imager is a type of normal-incidence telescope with a split thin metal filter, that is composed of aluminum/carbon $(\mathrm{Al} / \mathrm{C})$ and indium (In) in order to detect the resonance scattering emissions of helium ions (He II; $30.4 \mathrm{~nm}$ ) and oxygen ions (O II; $83.4 \mathrm{~nm}$ ). The primary scientific goals are: (1) to understand a global plasma distribution in the inner magnetosphere, and (2) to study the outflow mechanisms of oxygen ions from the polar ionosphere. The cold plasma in the plasmasphere also shows an active response at the high magnetic activity, which is contrary to a traditional understanding of plasmaspheric formation. Recent observations suggest that the heavy ions, such as oxygen ions, could also flow out. The remote sensing at $\mathrm{O}$ II by TEX is the first overall image of the world and will investigate the nature of the formation.

The optics in TEX consists of a multilayer-coated mirror to increase the reflectivity at $30.4 \mathrm{~nm}$, a split metallic thin filter, and microchannel plates to pick up photon events (Fig. 2). A high-voltage power supply (HVPS) is installed at one side, as shown in Fig. 2, and a preamplifier unit is contained along the other side. Survival heaters for the main body of the instrument, the HVPS, and the preamplifier (not shown in Fig. 3) are also installed, Table 1 summarizes
Table 1. Principal TEX design parameters.

\begin{tabular}{ll}
\hline Open Aperture & $48.6 \mathrm{~cm}^{2}$ \\
Format & Each $128 \times 64$ bins \\
FOV & Each 10.88×5.44 degree \\
Reflectivity of mirror & $18.8 \% @ 30.4 \mathrm{~nm} 20.2 \% @ 83.4 \mathrm{~nm}$ \\
Transmittance of filter & $11.2 \% @ 30.4 \mathrm{~nm} 2.1 \% @ 83.4 \mathrm{~nm}$ \\
Quantum efficiency of MCP & $7.6 \% @ 30.4 \mathrm{~nm} 9.8 \% @ 83.4 \mathrm{~nm}$ \\
Sensitivity & $0.014 \mathrm{cps} /$ Rayleigh at $30.4 \mathrm{~nm}$ \\
& $0.0036 \mathrm{cps} /$ Rayleigh at $83.4 \mathrm{~nm}$ \\
Prime focus & F/1.4 \\
Angular resolution & $0.085 \mathrm{degree}$ \\
Spatial resolution & $0.089 \mathrm{Re}$ \\
Maximum time resolution & $1 \mathrm{minute}$ \\
Mass & $2 \mathrm{~kg}$ \\
Size & $235 \mathrm{~mm} \times \phi 140 \mathrm{~mm}$ \\
\hline
\end{tabular}

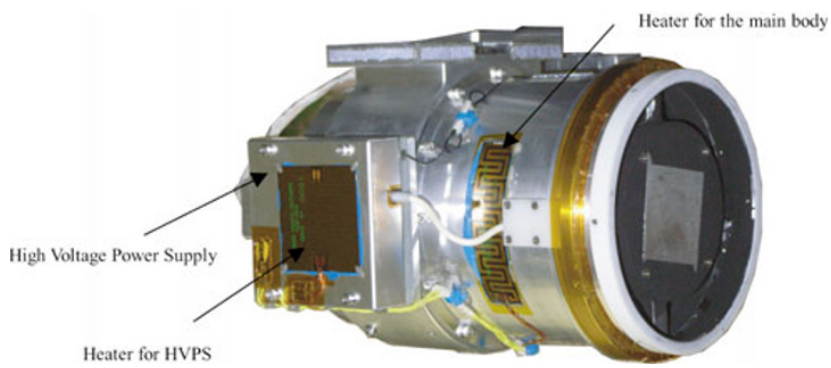

Fig. 3. Photograph of TEX without a multilayer thermal insulator. A high-voltage power supply is installed at this side. The preamplifiers are put at the opposite side.

the main features of TEX. The key points for the O II $(83.4 \mathrm{~nm})$ and He II $(30.4 \mathrm{~nm})$ imagings are (1) to eliminate the contamination from $\mathrm{H} \mathrm{I}(\mathrm{Ly}-\alpha)$, which is emitted from the geocorona at a maximum intensity of $10 \mathrm{kR}$ (Rairden et al., 1986) and (2) to enhance the detection efficiency at $30.4 \mathrm{~nm}$.

\subsection{Mo/Si multilayer-coated mirror}

In this section, we discuss the design and testing of a mirror coating. To realize a compact optics, we designed a multilayer-coated mirror for normal incidence reflection with a peak reflectivity at $30.4 \mathrm{~nm}$, because most materials have much higher single-layer reflectivities at $83.4 \mathrm{~nm}$. For example, the normal incidence reflectance of silicon at $83.4 \mathrm{~nm}$ is about $37 \%$. The mirror substrate is made of $\mathrm{Ze}-$ rodur, and the mirror has a focal length of $168 \mathrm{~mm}$, a diameter of $120 \mathrm{~mm}$, and a spherical shape to reduce aberration.

Our recent study found that a $\mathrm{Mg} / \mathrm{SiC}$ multilayer is a leading candidate for a highly reflective coating at $30.4 \mathrm{~nm}$ (Yoshikawa et al., 2005). We fabricated many samples and tested these to determine optical performance and degradation under conditions of vacuum and air. The $\mathrm{Mg} / \mathrm{SiC}$ coating has a high reflectivity $(>40 \%)$ at $30.4 \mathrm{~nm}$, but the $\mathrm{Mg} / \mathrm{SiC}$ stack is degraded even with only a short time exposure to the atmosphere (Murakami et al., 2006). Therefore, we decided to use a conventional Mo/Si multilayer coating in this mission. This decision was supported by the results of some successful space-borne experiments in which the Mo/Si multilayers had been used (Yoshikawa et al., 1997, 2001). For this space mission, we chose 20 alternating MoSi layers (period of $16.49 \mathrm{~nm}$, Mo thickness of $4.1 \mathrm{~nm}, \mathrm{Si}$ thickness of $12.39 \mathrm{~nm}$ ) on the Zerodur substrate of the mir- 


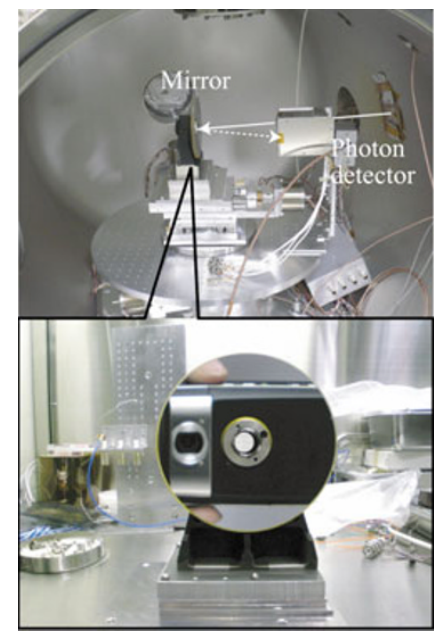

Fig. 4. Calibration setup in the EUV facility. Flight mirror was set up as so-called " $\theta-2 \theta$ " configuration in order to measure reflectivity against incident angles, points, and wavelength.

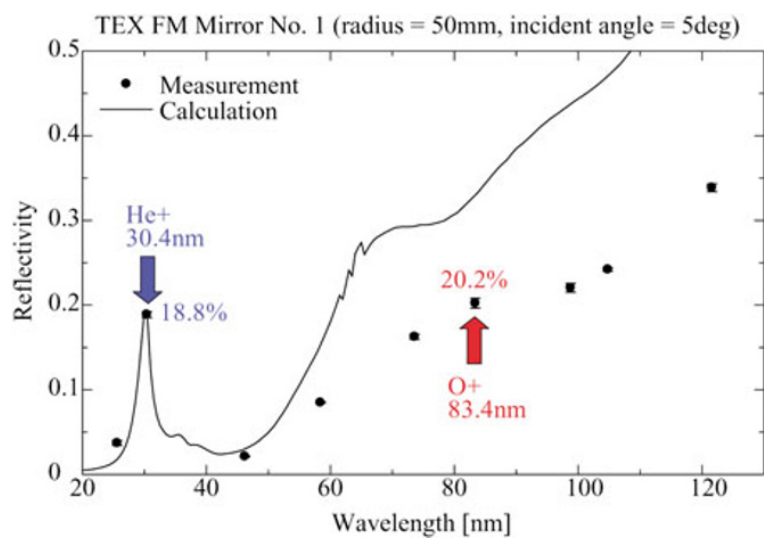

Fig. 5. Reflectivity of a Mo/Si multilayer coated mirror used in TEX. The reflectivity achieved up to $18.8 \%$. Unwanted longer wavelength emissions can be eliminated by a metallic thin filter.

ror. All layers were deposited by ion-beam sputtering. The Nikon Company manufactured the substrate and coating of the mirror.

The calibration system consists of a windowless RF excited flow lamp filled with source gases and connected to a JOVIN YVON LHT30 30-cm scanning monochromator. Using an atomic source with the monochromator provides radiation limited only by the line widths of spectral lines from the light source. In the vacuum chamber, the mirror was set on a manipulator with three orthogonal axes of translation and a single axis of rotation aligned perpendicularly to the incident beam. The incident and reflected beams were detected by a photon detector. The reflectivity was determined by measuring the ratio of reflected and incident beams. This facility has a capability of measuring the reflectance (and transmittance) at any position as a function of wavelength and incident angle. This process was repeated several times for each wavelength to enable us to compensate for long-term drifts in the source intensity. Figure 4 shows the calibration system in our EUV facility.

Figure 5 gives the average reflectivity of the flight mirror as a function of wavelength. We measured the reflectivities

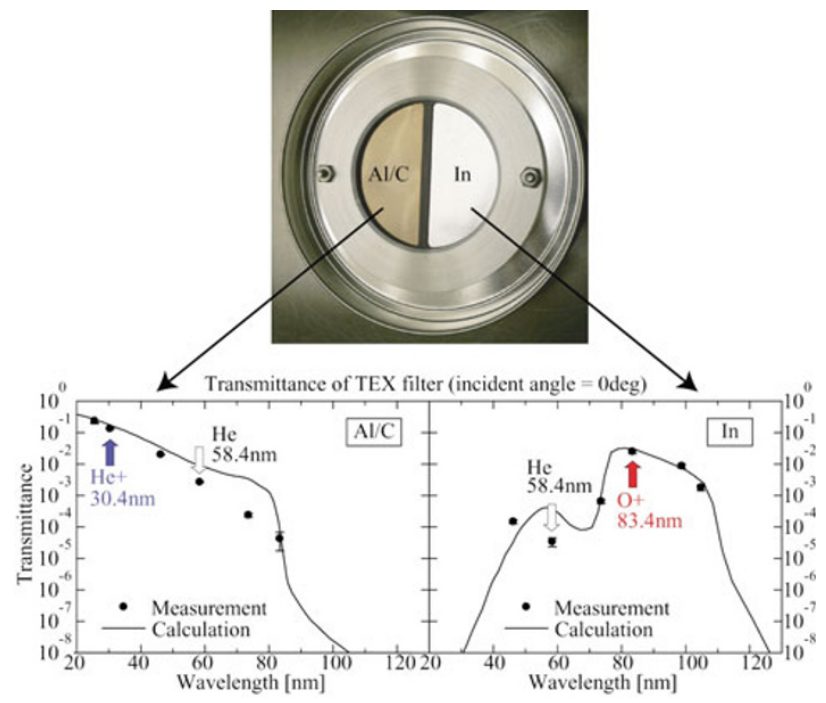

Fig. 6. Filter's transmittance. (Left) Aluminum (162.8 nm)/Carbon (31.2 $\mathrm{nm})$ part. This part is used for He II $(30.4 \mathrm{~nm})$ transmittance. (Right) Indium (292.9 nm) used for OII (34. $\mathrm{nm})$ transmittance.

at two points (30 and $50 \mathrm{~mm}$ from the center) toward every 90-degree radial direction using a $1-\mathrm{mm}$ diameter beam. The line in the figure represents a theoretical curve of $\mathrm{Mo} / \mathrm{Si}$ layers. At longer wavelengths, there is difference between the calculation and measurement, but this is due to the ambiguity of the optical parameter of Si in the EUV.

Although our testing of the mirrors shows that the Mo/Si multilayer coating was not particularly subject to degradation by oxidation, we protected the coating using normal procedures for handling. We stored the mirror in boxes purged by dry $\mathrm{N}_{2}$, and when the mirror had been installed in the telescope, we kept the overall instrument under as dry a $\mathrm{N}_{2}$ purge as practically possible.

\subsection{Metallic thin filter}

The filter material was chosen to provide high transmittance at the target wavelengths of 30.4 and $83.4 \mathrm{~nm}$, while attenuating the unwanted Ly- $\alpha(121.6 \mathrm{~nm})$ emission from the Earth's geocorona and the interplanetary medium. The filter is set at $12.3 \mathrm{~mm}$ off from the focal plane and is divided into two parts (Fig. 6). One part has a carbon coating of $31.2 \mathrm{~nm}$ on an Al film (162.8 nm), which attenuates emissions whose wavelengths are longer than $40 \mathrm{~nm}$; it allows the passage of He II $(30.4 \mathrm{~nm})$ with a small transmittance of He I $(58.4 \mathrm{~nm})$. The other part transmits O II $(83.4 \mathrm{~nm})$ and consists of a 292.9-nm thick layer of In. The filter is supported by a stainless steel mesh having a wire spacing of $0.36 \mathrm{~mm}$ and an open area of $75 \%$. The Al material was permitted to oxidize in air on one side, and In was oxidized on both sides. The oxidized coatings stabilize the material and provides the additional benefit of reducing the transmission of Ly- $\alpha$ (121.6 $\mathrm{nm})$.

Figure 6 (lower panels) compares the calibrated transmittances with theoretical ones. In the area of $\mathrm{Al} / \mathrm{C}$, carbon reduces transmittance at longer wavelengths, since $\mathrm{Al}$ itself has a fairly flat transmittance between 17 and $80 \mathrm{~nm}$. Oxidizing, as noted above, reduces the transmittance over all wavelengths, especially at longer wavelengths. The difference between theoretical and calibrated curves indicates the 


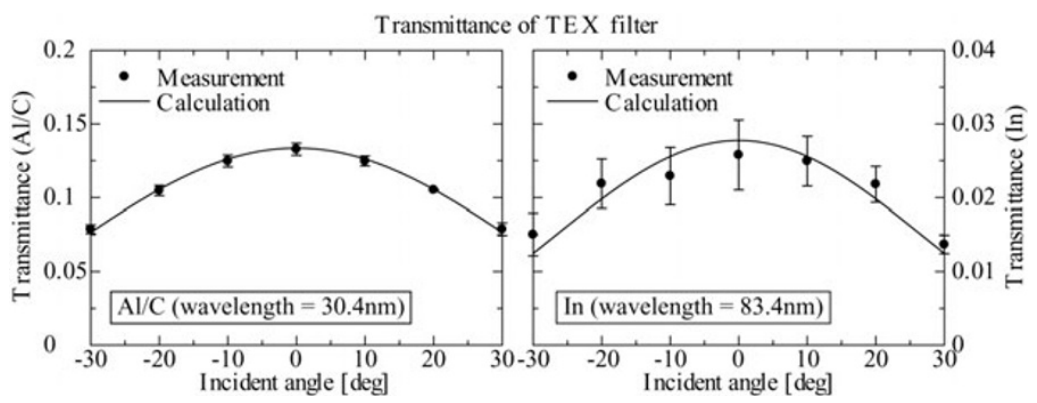

Fig. 7. Dependency of filter transmittance on incident angle.

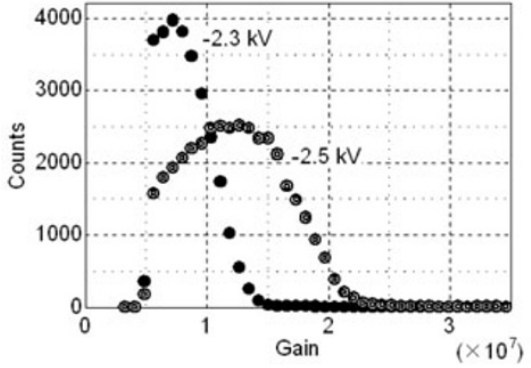

Fig. 8. Pulse-height distribution of MCPs with $-2.3 \mathrm{kV}$ and $-2.5 \mathrm{kV}$ applied. Pulse-height-ratio (PHR) of $96-106 \%$ and is achieved.

oxidization of the $\mathrm{Al}$ face. We conclude that transmittance above $120 \mathrm{~nm}$ is below $10^{-9}$, although no absolute calibrated value was available. The photon rejection at longer wavelengths is good enough to eliminate strong contamination from Earth's Ly- $\alpha$ geocorona. The In filter is used as band-pass filter to prevent He I, He II, and Ly- $\alpha$ emissions from contaminating O II emission. In particular, the Ly- $\alpha$ is so strong that the In filter is designed to be as thick as $292.9 \mathrm{~nm}$ despite low transmittance at $83.4 \mathrm{~nm}$. The right panel of Fig. 6 shows that the transmittance at Ly- $\alpha$ is $10^{6}$ fold lower than that at $83.4 \mathrm{~nm}$, but it is still sufficient to reduce the contamination of Ly- $\alpha$.

The dependence on the incident angle of the filter is shown in Fig. 7, together with the theoretical curves, showing good agreement with the theory.

\subsection{Microchannel plates (MCPs) and resistive anode equipment (RAE)}

We use three-stage MCPs for photon detection. Each MCP has a circular shape with an active area of $13.9 \mathrm{~cm}^{2}$, a bias angle of $8^{\circ}$, a $12-\mu \mathrm{m}$ pore size, an L/D (a ratio of length to pore diameter) of 40:1 and a total resistance of $125 \mathrm{MOhm}$. Three MCPs were placed in direct contact as a back-to-back stack. An important indicator of the MCP stack performance is the pulse height distribution of the single photon events. The gain and pulse height distribution (PHD) measurements were accomplished with a 30.4-nm illumination from the EUV lamp using the monochromator. Figure 8 shows the PHD with $-2.3 \mathrm{kV}$ and $-2.5 \mathrm{kV}$ applied at the MCP face. The pulse height ratio (PHR: width of the pulse height distribution) was somewhat broader$96 \%$ at $-2.3 \mathrm{kV}$ and $106 \%$ at $-2.5 \mathrm{kV}$-than we would expect from a five-stage MCP stack. The dark count is $0.28 \mathrm{cps} \mathrm{cm}^{-2}$ at $-2.3 \mathrm{kV}$ and $0.37 \mathrm{cps} \mathrm{cm}^{-2}$ at $-2.5 \mathrm{kV}$.

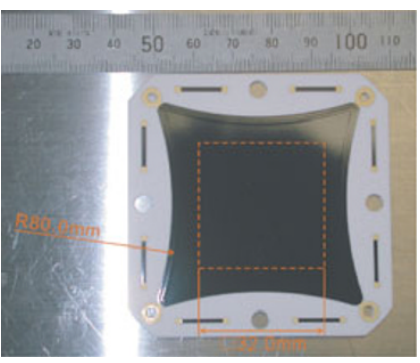

Fig. 9. Resistive Anode Equipment. Active area is $32 \mathrm{~mm} \times 32 \mathrm{~mm}$. Resistive face made of $\mathrm{RuO}_{2}$. We use $32 \mathrm{~mm} \times 32 \mathrm{~mm}$ at the center in order to avoid aberration near the edges.

Behind the MCPs, the resistive anode equipment is placed to analyze the position of photon injection (Fig. 9). The electron cloud emitted from the three-stage MCP consists of an order of $10^{6}$ electrons and impinges on RAE. The electron cloud is divided over four electrodes at the corners in reverse proportional to the distance to them. Image distortion in the RAE arises mainly because the MCP electron clouds at the edge run off the field. Therefore, we use an active area of $32 \times 32 \mathrm{~mm}^{2}$ e on the RAE to avoid the distortion.

Figure 10 shows the block diagram of a position analysis in TEX electronics. The signal at each electrode is amplified by A-225 (AMPTEK). The pulse-shaper (A-206) further amplifies the signal and recognizes the event with a threshold level. When A-206 identifies a significant signal level, it sends a "ready" signal to the main electronics. PH300 holds the signal peak and ADC digitalizes the height of signal. The main electronics computes the position.

The image linearity and resolution were measured by imaging an array of pinholes in a metal mask illuminated by $30.4 \mathrm{~nm}$ beam, as shown in Fig. 11. The ion-etching mask was placed at $8.3 \mathrm{~mm}$ off from the input face. The array consists of $0.5 \mathrm{~mm}$ or $1.0 \mathrm{~mm}$ holes with 5 - $\mathrm{mm}$ distance. The pinhole mask image (left panel) clarifies none of distortion of the image within active area of $32-\mathrm{mm} \times$ 32-mm. Right panel is a cross-section of the left panel in the red line, indicates $250-\mu \mathrm{m}$ resolution is achieved.

\subsection{Electronics and high-voltage power supply}

$\mathrm{SH}-3$ (Hitachi) controls the equatorial mounting gimbal system and the data interface to two telescopes as well as recognizes commands from command receiver. The highvoltage power supply installed at the side of TEX is turned on by commands from $\mathrm{SH}-3$. 


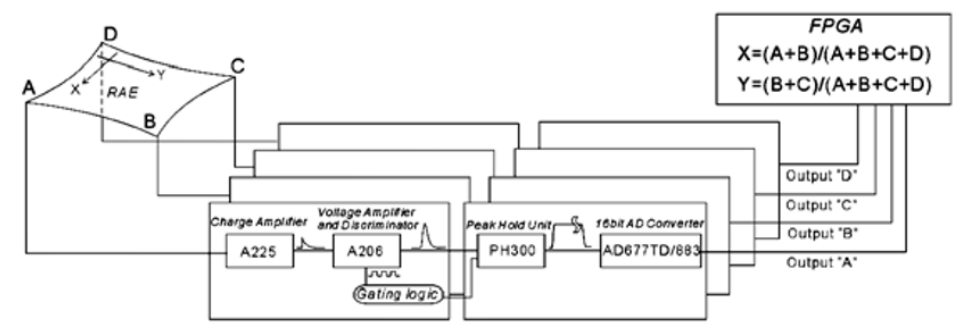

Fig. 10. Block diagram of position analysis electronics. Preamplifiers, pulse-shapers with discriminators, and peak-hold ICs are all AMPTEK Co. products. This system realizes 128-bin (resolution) for each axis.
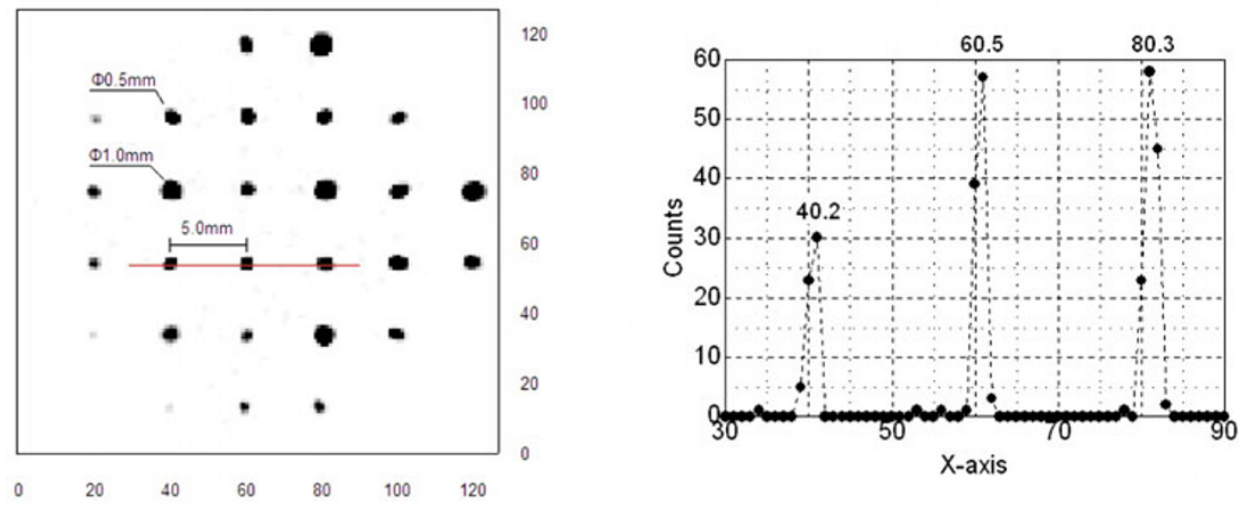

Fig. 11. (Left) Pinhole mask image obtained by the TEX detector. We illuminated $30.4 \mathrm{~nm}$ beam onto the mask. Distance between holes is $5 \mathrm{~mm}$, hole's diameters are $0.5 \mathrm{~mm}$ or $1.0 \mathrm{~mm}$. (Right) Cross-section of red line in the left panel. Resolution of $32 \mathrm{~mm} / 128(250 \mu \mathrm{m})$ is achieved.

\subsection{Total sensitivity}

The TEX has a full circular field-of-view (FOV) of $10^{\circ}$, and the $128 \times 128$ bins corresponding to the spatial resolution of $0.09 \mathrm{Re}$ on the Earth's surface from the lunar orbit. One-half of the FOV $(128 \times 64$ bins $)$ is used for O II $(83.4 \mathrm{~nm})$ detection; the other is for He II $(30.4 \mathrm{~nm})$.

The signal count rate $N$ (cps) of the emission is represented by the following equations,

$$
N=10^{6} / 4 \pi \cdot I \cdot S \cdot \Delta \cdot T_{\text {Filter }} \cdot R_{\text {mirror }} \cdot E_{\mathrm{MCP}}[\mathrm{cps}],
$$

where $I$ is the brightness of emission in (Rayleigh), $S$ is an entrance aperture $\left(\mathrm{cm}^{2}\right), \Delta$ is the sold angle of identical FOV, $T_{\text {Filter }}$ is transmittance, $R_{\text {mirror }}$ is a mirror's reflectivity, and $E_{\mathrm{MCP}}$ is a quantum efficiency of MCP. Those instrumental parameters are summarized in Table 1 . The telescope has a total efficiency of $0.014 \mathrm{cps} \mathrm{R}^{-1}$ for the He II $(30.4 \mathrm{~nm})$ bin and $0.0036 \mathrm{cps} \mathrm{R}^{-1}$ for the O II $(83.4 \mathrm{~nm})$ bin.

In the following, we estimate the signal-to-noise ratio (SNR) during observation. The detector behaves as an ideal photon counter so that signal corresponds to the number of photon events collected during an exposure period $\left(t_{\exp }\right)$, and noise is the square root of signal.

Instrumental background (noise count from MCPs in the detector unit) is a primary noise source. The MCPs manufactured by Hamamatsu Photonics Co. had a noise count by $10.4 \mathrm{cps} \mathrm{cm}^{-2}$ in our previous planetary mission, although the noise count in the laboratory was below $1 \mathrm{cps} \mathrm{cm}^{-2}$ (Yoshikawa et al., 2000a; Yamazaki et al., 2006). This increase was due to cosmic ray and/or high energy particle bombardments in the magnetosphere. For the lunar environment, we assume the latter component is negligible but as the worst case analysis, we assume $10.4 \mathrm{cps} \mathrm{cm}^{-2}(0.0065$ cps bin $^{-1}$ ) as dark count in lunar orbiter.

The second noise source is a contamination from He I (58.4 $\mathrm{nm})$ and Ly- $\alpha$ emissions. As stated above, Ly- $\alpha$ is sufficiently reduced by the metallic filter on both sides. The intensity of Ly- $\alpha$ is insensitive to our estimation of SNR. In the following discussion, we assumed and fixed the Ly- $\alpha$ intensity at $5 \mathrm{kR}$. Another possible contamination is He I $(58.4 \mathrm{~nm})$ emission. This is fairly well reduced by the filter, but it has the potential to contaminate our observation. We assume the maximum geocoronal $\mathrm{He} \mathrm{I}(58.4 \mathrm{~nm})$ intensity to be $500 \mathrm{R}$ (Meier, 1991) and interstellar He I (58.4 nm) to be 10 R (Yamazaki et al., 2002).

During the measurement, a detector pixel sees the EUV signal $\left(N_{\text {Signal }}\right)$, measured in cps bin ${ }^{-1}$, and the background ( $N_{\text {Background }}$ instrumental background plus contaminations from the other EUV emissions), measured in the same units. The number of signal counts is the total observed counts minus the background

$$
\begin{aligned}
N_{\text {Total }} & =N_{\text {Signal }}+N_{\text {Background }} \\
N_{\text {Signal }} & =N_{\text {Total }}-N_{\text {Background }} \\
\sigma_{\text {Signal }} & =\sqrt{\sigma_{\text {Total }}^{2}+\sigma_{\text {Background }}^{2}} \approx \sqrt{\sigma_{\text {Signal }}^{2}+2 \cdot \sigma_{\text {Background }}^{2}} \\
& \approx \sqrt{N_{\text {Signal }}+2 \cdot N_{\text {Background }}} \\
\text { SNR } & =\frac{N_{\text {Signal }}}{\sigma_{\text {Signal }}}=\frac{N_{\text {Signal }}}{\sqrt{N_{\text {Signal }}+2 \cdot N_{\text {Background }}}}
\end{aligned}
$$



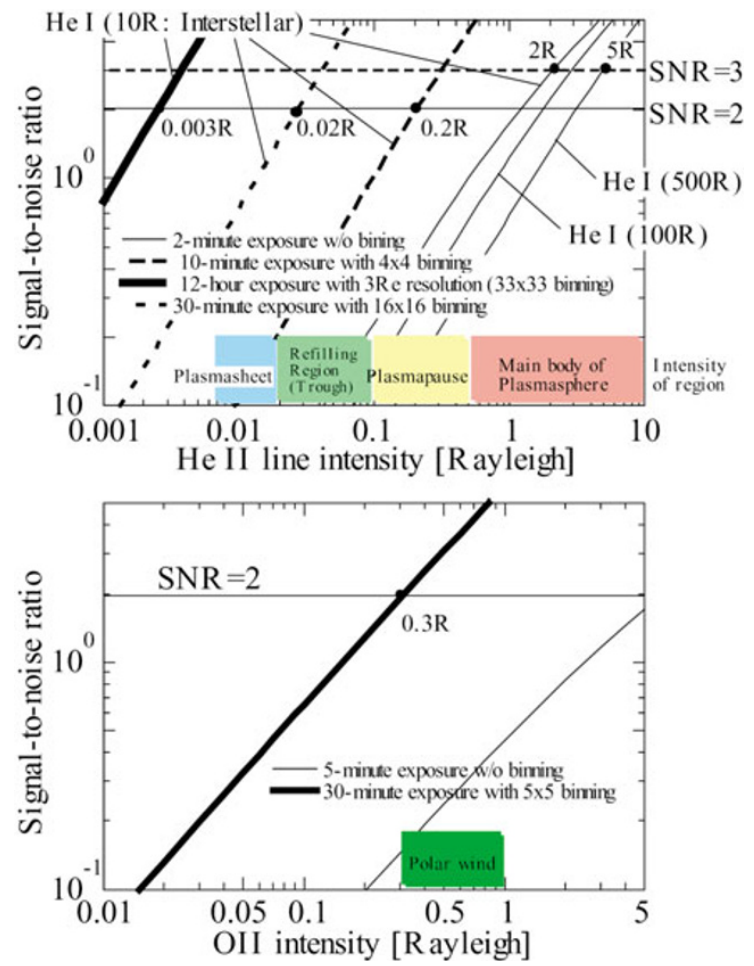

Fig. 12. (Upper panel) Expected Signal-to-Noise Ratios for He II (30.4 nm) observation. The SNR is insensitive to ionospheric contamination at $121.6 \mathrm{~nm}$. Here we assume Ly- $\alpha$ intensity to be $5 \mathrm{kR}$. The intensity of He I ( $58.4 \mathrm{~nm}$ ) is assumed to be $500 \mathrm{R}, 100 \mathrm{R}$ (ionosphere) and $10 \mathrm{R}$ (interstellar) as indicated. (Lower panel) Expected SNR for O II $(83.4 \mathrm{~nm})$ observation. The observation is free of ionospheric He I and $\mathrm{Ly}-\alpha$ contaminations in the range of $10-500 \mathrm{R}$ for $\mathrm{He} \mathrm{I}(58.4 \mathrm{~nm})$ and $1 \mathrm{k}-30 \mathrm{kR}$ for $\mathrm{Ly}-\alpha$. In this plot, the intensities of $\mathrm{He} \mathrm{I}$ and $\mathrm{Ly}-\alpha$ are $100 \mathrm{R}$ and $5 \mathrm{kR}$, respectively.

Figure 12 shows SNRs based on the above assumptions. Expected intensities discussed in Section 2 are also indicated. Here we set "SNR=2 or 3" as a norm. For He II (30.4 nm) observation, the observation mode (2-min exposure and $0.09 \mathrm{Re}$ resolution) is able to identify the main body of the plasmasphere with a high reliability $(\mathrm{SNR}=3)$. The identifications of plasmapause and refilling region in the trough discussed in Section 2 are feasible with a fairly good SNR. Imaging of the Plasmasheet is the most challenging target, but we will be able to achieve the science goal against the low sky background of He I (10 R).

Imaging polar wind by O II $(83.4 \mathrm{~nm})$ is also challenging target, but the 30-min exposure with $0.5 \mathrm{Re}$ resolution allows the polar wind to be visible with $\mathrm{SNR}=2$.

\section{Summary}

We will image near-Earth cold plasmas at O II (83.4 nm) and He II $(30.4 \mathrm{~nm})$ emissions using the UPI-TEX instrument aboard the SELENE satellite. The UPI-TEX instrument has enough performance to detect the oxygen ion outflow, the transport route from the polar ionosphere into the magnetosphere, and the plasmasphere. The 2-D O II imagery is expected to identify the transport routes and mechanisms of the cold $\mathrm{O}^{+}$ions and to reveal a quantitative balance between the supply and loss, including thermal plasma. On the other hand, the detection of He II $(30.4 \mathrm{~nm})$ by the TEX instrument is devoted to the new challenge of imaging the magnetosphere (plasmasheet) as well as to contributing to conventional plasmasphere physics. The instrumental specification is listed in Table 1.

Acknowledgments. The authors thank all of the members of the SELENE project team for their effort in achieving a successful mission.

\section{References}

Abe, T., B. A. Whalen, A. W. Yau, R. E. Horita, S. Watanabe, and E. Sagawa, EXOS D (Akebono) suprathermal mass spectrometer observations of the polar wind, J. Geophys. Res., 98, 11,191, 1993.

Abe, T., S. Watanabe, B. A. Whalen, A. W. Yau, and E. Sagawa, Observations of polar wind and thermal ion outflow by Akebono/SMS, J. Geomag. Geoelectr., 48, 319, 1996.

Axford, W. I., The polar wind and the terrestrial helium budget, J. Geophys. Res., 73, 6855, 1968

Banks, P. M. and T. E. Holzer, The polar wind, J. Geophys. Res., 73, 6846, 1968.

Banks, P. M., A. F. Nagy, and W. I. Axford, Dynamical behavior of thermal protons in the mid-latitude ionosphere and magnetosphere, Planet. Space Sci., 19, 1053, 1971.

Burch, J. L., S. B. Mende, D. G. Mitchell, T. E. Moore, C. J. Pollock, B. W. Reinisch, B. R. Sandel, S. A. Fuselier, D. L. Gallagher, J. L. Green, J. D. Perez, and P. H. Reiff, Views of earth's magnetosphere with the IMAGE satellite, Science, 291, 619, 2001a.

Burch, J. L., D. G. Mitchell, B. R. Sandel, P. C. Brandt, and M. Wüest, Global dynamics of the plasmasphere and ring current during magnetic storms, Geophys. Res. Lett., 28, 1159, 2001b.

Chakrabarti, S., Magnetospheric Imagery and Atmospheric Remote Sensing, Optic. Eng., 32, 3007, 1993.

Chakrabarti, S., F. Paresce, C. S. Bowyer, Y. T. Chiu, and A. Aikin, Plasmaspheric helium ion distribution from satellite observations of $\mathrm{He}$ II 304A, Geophys. Res. Lett., 9, 151, 1982.

Chandler, M. O., J. H. Waite Jr., and T. E. Moore, Observations of polar ion outflows, J. Geophys. Res., 96, 1421, 1991.

Chappell, C. R., The convergence of fact and theory on magnetospheric convection, Astrophys. Space Sci. Lib., 42, 277, 1974.

Chappell, C. R., K. K. Harris, and G. W. Sharp, The relationship of the plasmasphere and the stable auroral red arcs in the magnetic storm of october 29 to november 7, 1968, Astrophys. Space Sci. Lib., 24, 73, 1971.

Chappell, C. R., J. H. Waite Jr., and T. E. Moore, The ionosphere as a fully adequate source of plasma for the Earth's magnetosphere, J. Geophys. Res., 92, 5896, 1987.

Chen, A. J. and R. A. Wolf, Effects on the plasmasphere of a time-varying convection electric field, Planet. Space Sci., 20, 483, 1972.

Chiu, Y. T., R. M. Robinson, G. R. Swenson, S. Chakrabarti, and D. S. Evans, Imaging the outflow of ionospheric ions into the magnetosphere, Nature, 322, 441, 1986.

Chiu, Y. T., R. M. Robinson, and H. L. Colin, Magnetospheric and exospheric imaging in the extreme ultraviolet, Geophys. Res. Lett., 17, 267, 1990.

Collin, H. L., R. D. Sharp, and E. G. Shelley, The magnitude and composition of the outflow of energetic ions from the ionosphere, J. Geophys. Res., 89, 2185, 1984.

Cotton, D. M., R. Conant, and S. Chakrabarti, Wide-angle geocoronal telescope: a He-II 304-A plasmaspheric imager, Optic. Eng., 32, 3170, 1993.

Cowley, S. W. H., Plasma populations in a simple open model magnetosphere, Space Sci.Rev., 26, 217, 1980.

Fuselier, S. A., A. G. Ghielmetti, T. E. Moore, M. R. Collier, J. M. Quinn, G. R. Wilson, P. Wurz, S. B. Mende, H. U. Frey, C. Jamar, J.-C. Gerard, and J. L. Burch, Ion outflow observed by IMAGE: Implications for source regions and heating mechanisms, Geophys. Res. Lett., 28, 1163, 2001.

Grebowsky, J. M., Time Dependent plasmapause motion, J. Geophys. Res, 75, 4329, 1971.

Hashimoto, K. K., T. Kikuchi, and Y. Ebihara, Response of the magnetospheric convection to sudden interplanetary magnetic field changes as deduced from the evolution of partial ring currents, J. Geophys. Res., 107(A11), 1337, 2002.

Hirahara, M., T. Mukai, T. Terasawa, S. Machida, Y. Saito, T. Yamamoto, and S. Kokubun, Cold dense ion flows with multiple components ob- 
served in the distant tail lobe by Geotail, J. Geophys. Res., 101, 7769, 1996.

Kikuchi, T., H. Luhr, T. Kitamura, O. Saka, and K. Schlegel, Direct penetration of the polar electric field to the equator during a DP2 event as detected by the auroral and equatorial magnetometer chains and the EISCAT radar, J. Geophys. Res., 101, 17,161, 1996.

Lennartsson, W. and E. G. Shelley, Survey of 0.1- to 16-keV/e plasma sheet ion composition, J. Geophys. Res., 91, 3061, 1986.

Lu, G., T. E. Holzer, D. Lummerzheim, J. M. Ruohoniemi, P. Stauning, O. Troshichev, P. T. Newell, M. Brittnacher, and G. Parks, Ionospheric response to the inter-planetary magnetic field southward turning: Fast onset and slow reconfiguration, J. Geophys. Res., 107(A8), 1153, 2002.

Lyons, L. R. and T. W. Speiser, Evidence for current sheet acceleration in the geomagnetic tail, J. Geophys. Res., 87, 2276, 1982.

Matsui, H., T. Mukai, S. Ohtani, K. Hayashi, R. C. Elphic, M. F. Thomsen, and $\mathrm{H}$. Matsumoto, Cold dense plasma in the outer magnetosphere, $J$. Geophys. Res., 104, 25077, 1999.

Meier, R. R., Ultraviolet spectroscopy and remote sensing of the upper atmosphere, Space Sci. Rev., 58, 1, 1991.

Meier, R. R. and C. S. Weller, EUV resonance radiation from helium atoms and ions in the geocorona, J. Geophys. Res., 77, 1190, 1972

Moldwin, M. B., M. F. Thomsen, S. J. Bame, D. J. McComas, and K. R. Moore, An examination of the structure and dynamics of the outer plasmasphere using multiple geosynchronous satellites, J. Geophys. Res., 99, 11475, 1994

Moore, T. E., D. J. Chornay, M. R. Collier, F. A. Herrero, J. Johnson, M. A. Johnson, J. W. Keller, J. F. Laudadio, J. F. Lobell, K. W. Ogilvie, P. Rozmarynowski, S. A. Fuselier, A. G. Ghielmetti, E. Hertzberg, D. C. Hamilton, R. Lundgren, P. Wilson, P. Walpole, T. M. Stephen, B. L. Peko, B. Van Zyl, P. Wurz, J. M. Quinn, and G. R. Wilson, The low energy neutral atom imager for IMAGE, Space Sci.Rev., 91, 155, 2000.

Moore, T. E., M. R. Collier, J. L. Burch, D. J. Chornay, S. A. Fuselier, A. G. Ghielmetti, B. L. Giles, D. C. Hamilton, F. A. Herrero, J. W. Keller, K. W. Ogilvie, B. L. Peko, J. M. Quinn, T. M. Stephen, G. R. Wilson, and P. Wurz, Low energy neutral atoms in the magnetosphere, Geophys. Res. Lett., 28, 1143, 2001.

Mukai, T., M. Hirahara, S. Machida, Y. Saito, T. Terasawa, and A. Nishida, Geotail observation of cold ion streams in the medium distance magnetotail lobe in the course of a substorm, Geophys. Res. Lett., 21, 1023, 1994.

Murakami, G., K. Yoshioka, and I. Yoshikawa, Development of Mg/SiC multilayer mirrors, Proceeding of SPIE, 6317, 631714-1, 2006.

Murakami, G., M. Hirai, and I. Yoshikawa, The plasmapause response to the southward turning of the IMF derived from sequential EUV images, J. Geophys. Res., 112, A06217, doi:10.1029/2006JA012174, 2007.

Nakamura, M., I. Yoshikawa, A. Yamazaki, K. Shiomi, Y. Takizawa, M. Hirahara, K. Yamashita, Y. Saito, and W. Miyake, Terrestrial plasmaspheric imaging by an extreme ultraviolet scanner on Planet-B, Geophys. Res. Lett., 27, 141, 2000.

Nishida, A., Formation of plasmapause, or magnetospheric plasma knee, by the combined action of magnetospheric convection and plasma escape from the tail, J. Geophys. Res., 71, 5669, 1966.

Obara, T., M. Den, Y. Miyoshi, and A. Morioka, Energetic electron variation in the outer radiation zone during early May 1998 magnetic storm, J. Atmos. Solar-Terr. Phys., 62, 1405, 2000.

Ogawa, T. and T. Thomatsu, Sounding rocket observation of helium 304 and 584-A glow, J. Geophys. Res., 76, 6136, 1971.

Ondoh, T. and K. Aikyo, Effects of tailward stretching geomagnetic field on drift motion of plasma particles in the magnetospheric equatorial plane, Adv. Space Res., 6, 203, 1986.

Paresce, F., C. S. Bowyer, and S. Kumar, On the distribution of $\mathrm{He}^{+}$in the plasmasphere from observations of resonantly scattered He II 304A radiation, J. Geophys. Res., 79, 174, 1974.

Park, C. G., Whistler observations of the interchange of ionization between the ionosphere and the protonosphere, J. Geophys. Res., 75, 4249, 1970.

Rairden, R. L., L. A. Frank, and J. D. Craven, Geocoronal imaging with Dynamics Explorer, J. Geophys. Res., 91, 13613, 1986.

Ridley, A. J., G. Lu, C. R. Clauer, and V. O. Papitashvili, A statistical study of the ionospheric convection response to changing interplanetary magnetic field conditions using the assimilative mapping of ionospheric electrodynamics technique, J. Geophys. Res., 103(A3), 4023, 1998.

Roelof, E. C., B. H. Mauk, Barry, and R. R. Meier, Instrument requirements for imaging the magnetosphere in extreme ultraviolet and en- ergetic neutral atoms derived from computer-simulated images, Proc. SPIE, 1744, 19, 1992.

Ruohoniemi, J. M. and R. A. Greenwald, The response of high-latitude convection to a sudden southward IMF turning, Geophys. Res. Lett., 25, 2913, 1998.

Saito, Y., T. Mukai, M. Hirahara, S. Machida, A. Nishida, T. Terasawa, S. Kokubun, and T. Yamamoto, GEOTAIL observation of ring-shaped ion distribution functions in the plasma sheet-lobe boundary, Geophys. Res. Lett., 21, 2999, 1994.

Sandel, B. R., R. A. King, W. T. Forrester, D. L. Gallagher, A. L. Broadfoot, and C. C. Curtis, Initial results from the IMAGE extreme ultraviolet imager, Geophys. Res. Lett., 28, 1439, 2001.

Schulz, M. and H. C. Koons, Thermalization of colliding ion streams beyond the plasmapause, J. Geophys. Res., 77, 248, 1972.

Seki, K., M. Hirahara, T. Terasawa, I. Shinohara, T. Mukai, Y. Saito, S. Machida, and T. Yamamoto, Coexistence of Earth-origin $\mathrm{O}^{+}$and solar wind-origin $\mathrm{H}^{+} / \mathrm{He}^{++}$in the distant magnetotail, Geophys. Res. Lett., 23, 985, 1996.

Seki, K., M. Hirahara, T. Terasawa, T. Mukai, Y. Saito, S. Machida, T. Yamamoto, and S. Kokubun, Statistical properties and possible supply mechanisms of tailward cold $\mathrm{O}^{+}$beams in the lobe/mantle regions, $J$. Geophys. Res., 103, 4477, 1998.

Seki, K., M. Hirahara, T. Terasawa, T. Mukai, and S. Kokubun, Properties of $\mathrm{He}^{+}$beams observed by Geotail in the lobe/mantle regions: Comparison with $\mathrm{O}^{+}$beams, J. Geophys. Res., 104, 6973, 1999.

Seki, K., R. C. Elphic, M. F. Thomsen, J. Bonnell, E. J. Lund, M. Hirahara, T. Terasawa, and T. Mukai, Cold flowing $\mathrm{O}^{+}$beams in the lobe/mantle at Geotail: Does FAST observe the source?, J. Geophys. Res., 105, 15931, 2000.

Seki, K., R. C. Elphic, M. Hirahara, T. Terasawa, and T. Mukai, On atmospheric loss of oxygen ions from earth through magnetospheric processes, Science, 291, 1939, 2001.

Seki, K., M. Hirahara, T. Terasawa, T. Mukai, and R. Elphic, Dynamics of oxygen ions in the Earth s magnetotail: Geotail and FAST observations, 34th COSPAR Scientific Assembly, 2002.

Wilson, G. R., J. L. Horwitz, and J. Lin, A semikinetic model for early stage plasmasphere refilling. I-Effects of Coulomb collisions, J. Geophys. Res., 97, 1109, 1992.

Yamazaki, A., S. Tashiro, Y. Nakasaka, I. Yoshikawa, W. Miyake, and M. Nakamura, Sounding-rocket observation of O II 83.4-nm emission over the polar ionosphere, Geophys. Res. Lett., 29, 2005, 2002.

Yamazaki, A., I. Yoshikawa, K. Shiomi, Y. Takizawa, W. Miyake, and M. Nakamura, Latitudinal variation of the solar He I $58.4 \mathrm{~nm}$ irradiance from the optical observation of the interplanetary He I emission, $J$. Geophys. Res., 111(A6), CiteID A06106, 2006.

Yau, A. W., P. H. Beckwith, W. K. Peterson, and E. G. Shelley, Long-term (solar cycle) and seasonal variations of upflowing ionospheric ion events at DE 1 altitudes, J. Geophys. Res., 90, 6395, 1985.

Yoshikawa, I., M. Nakamura, M. Hirahara, Y. Takizawa, K. Yamashita. H. Kunieda, T. Yamazaki, K. Misaki, and A. Yamaguchi, Observation of He II emission from the plasmasphere by a newly developed EUV telescope on board sounding rocket S-520-19, J. Geophys. Res., 109, 19897, 1997.

Yoshikawa, I., A. Yamazaki, K. Shiomi, K. Yamashita, Y. Takizawa, and M. Nakamura, Evolution of the outer plasmasphere during low geomagnetic activity observed by the EUV scanner onboard Planet-B, J. Geophys. Res., 105, 27777, 2000a.

Yoshikawa, I., A. Yamazaki, K. Shiomi, K. Yamashita, Y. Takizawa, and M. Nakamura, Photometric measurement of cold helium ions in the magnetotail by an EUV scanner onboard Planet-B: Evidence of the existence of cold plasmas in the near-Earth plasma sheet, Geophys. Res. Lett., 27, 3567, 2000b.

Yoshikawa, I., A. Yamazaki, K. Shiomi, M. Nakamura, K. Yamashita, Y. Saito, M. Hirahara, Y. Takizawa, W. Miyake, and S. Matsuura, Development of a compact EUV photometer for imaging the planetary magnetosphere, J. Geophys. Res., 106, 26057, 2001.

Yoshikawa, I., T. Murachi, H. Takenaka, and S. Ichimaru, Multilayer coating for $30.4 \mathrm{~nm}$, Rev. Sci. Inst., 76, Issue 6, pp. 066109-066109-2, 2005.

I. Yoshikawa (e-mail: yoshikawa@eps.s.u-tokyo.ac.jp), A. Yamazaki, G. Murakami, K. Yoshioka, S. Kameda, F. Ezawa, T. Toyota, W. Miyake, M. Taguchi, M. Kikuchi, and M. Nakamura 